\title{
Terminologie outillée et diachronie : éléments de réflexion autour d'une réconciliation
}

Textual terminology and diachrony: reflections about a reconciliation

\section{Aurélie Picton}

\section{OpenEdition}

\section{Journals}

Édition électronique

URL : http://journals.openedition.org/asp/5255

DOI : $10.4000 /$ asp. 5255

ISSN : 2108-6354

\section{Éditeur}

Groupe d'étude et de recherche en anglais de spécialité

Édition imprimée

Date de publication : 1 novembre 2018

Pagination : 27-52

ISSN : 1246-8185

\section{Référence électronique}

Aurélie Picton, «Terminologie outillée et diachronie : éléments de réflexion autour d'une réconciliation », ASp [En ligne], 74 | 2018, mis en ligne le 01 novembre 2019, consulté le 01 novembre 2020. URL : http://journals.openedition.org/asp/5255 ; DOI : https://doi.org/10.4000/asp.5255

Ce document a été généré automatiquement le 1 novembre 2020

Tous droits réservés 


\title{
Terminologie outillée et diachronie : éléments de réflexion autour d'une réconciliation
}

Textual terminology and diachrony: reflections about a reconciliation

\author{
Aurélie Picton
}

\section{Réconciliation entre diachronie et langues de spécialité}

1 En langues de spécialité (LSP), la dimension diachronique a longtemps été considérée comme le parent pauvre des recherches scientifiques (voir par exemple Dury \& Picton 2008 ; Humbley 2011; Møller 1998; Van der Yeught 2009). Ce constat s'explique en partie par une histoire théorique très contrastée, en particulier en comparaison avec l'histoire de la linguistique, marquée quant à elle par la primauté des études historiques :

La seule étude scientifique du langage est la méthode historique [...]. Toute étude linguistique scientifique qui n'est pas historique dans ses buts ni dans ses méthodes peut s'expliquer seulement soit par une déficience du chercheur, soit par l'insuffisance des sources dont il dispose. (Hermann Paul, Principien der Sprachgeschishte, 1880, tr. P. Caussat). (cité dans Normand [2000:30])

2 En terminologie, la perspective wüsterienne (ou Théorie générale de la terminologie [TGT]), longtemps dominante, préconisait en effet une vision prescriptive, limitant ainsi la prise en compte de l'évolution à la création consciente de termes par les experts (Wüster 1981 : 63). Cette vision, de fait, a éloigné la dimension diachronique.

3 Face aux réalités des langues de spécialité en discours, et en réponse aux limites imposées par cette vision ${ }^{1}$, plusieurs propositions théoriques ont vu le jour (Bourigault \& Slodzian 1999; Cabré 1998; Gaudin 1993 ; 2003; Temmerman 2000; Desmet 2007, etc.) qui, toutes, plaident en faveur d'un rapprochement avec la linguistique, et de la prise en compte de la polysémie et de la variation des termes, en corpus. Avec ce 
changement de paradigme amorcé dans les années 1990, la diachronie a repris sa légitimité, mais a gardé longtemps, malgré tout, une place relativement confidentielle.

Avant ce changement de paradigme, cette perspective n'est pas totalement absente (Candel 2004) et différents points de vue ont été adoptés sur cette question. L'on peut notamment souligner les recherches développant la diachronie comme une « motivation » envers une certaine vision prescriptive (Wüster 1981 : 63-65). Ou encore, plusieurs études ad hoc de l'évolution de terminologies ont été proposées par des lexicologues ou des experts (par ex. Auger 1973; Quemada 1978 ; Sournia 1994).

Néanmoins, la fin des années 1990 a marqué le début d'une dynamique différente où nombre de travaux ont alors pris la forme de véritables plaidoyers en faveur de sa prise en compte (notamment Dury 1997; Dury 2013; Dury \& Picton 2008; Humbley 2011 ; Møller 1994 ; 1998 ; Van der Yeught 2009). Dans cette continuité, depuis les années 2000 , de plus en plus de travaux et recherches voient le jour, autour de la question de la diachronie en langues de spécialité (Banks 2012 ; Candel \& Gaudin 2006; Charpy 2011 ; Dury 2013; Lafrance 2016; Magnet 2000; Peruzzo 2013; Picton 2009; Picton 2014 ; Wozniak 2015, pour n'en citer que quelques-uns).

6 Ces travaux présentent néanmoins une grande hétérogénéité tant dans leurs approches que dans leurs objectifs : certains auteurs comparent des corpus, d'autres comparent des dictionnaires, des corpus et des dictionnaires, de manière plus ou moins automatisée, pour observer des termes, des concepts, des constructions syntaxiques, des genres, etc. Du point de vue temporel, les études existantes se penchent aussi bien sur des intervalles longs, de plusieurs dizaines d'années que sur des approches en diachronie courte, de moins de dix ou vingt ans. Enfin, le point de vue adopté par ces différents travaux peut concerner tout aussi bien les évolutions d'un système linguistique que l'évolution des connaissances, l'histoire d'une discipline, d'une période contemporaine ou historique, etc.

7 Si ce constat reflète la richesse des apports de la perspective diachronique et une réconciliation en cours, il indique également que l'heure semble moins au plaidoyer en faveur de la diachronie, qu'à la réflexion autour de ce qui constitue la perspective diachronique lorsqu'elle est appliquée aux langues de spécialité.

\section{2. Étapes de cette réconciliation}

8 Dans un article paru en 2009, et écrit avec Pascaline Dury, quatre types d'obstacles à la réconciliation entre diachronie et LSP avaient été identifiés :

1. des obstacles d'ordre théorique, en lien avec la prédominance de la théorie classique en terminologie, normative et synchronique (cf. supra);

2. des obstacles d'ordre méthodologique, liés à la difficulté de compilation des corpus diachroniques équilibrés et à l'accès à des outils adéquats pour explorer ce type de corpus comparables ;

3. des obstacles d'ordre pragmatique, qui renvoient au fait que les intérêts de la dimension diachronique pour l'analyse sont certainement, en partie du moins, moins perceptibles que ceux de la dimension synchronique et donc moins facilement financés ;

4. des obstacles d'ordre "psychologiques », relatifs au manque de réflexion théorique de fond sur ce qui constitue la diachronie en langues de spécialité. 

publication de cet article, en grande partie levés. En effet, comme nous l'avons souligné, les théories actuelles font la part belle à la variation, à la diachronie et aux corpus. La constitution de ces derniers est, par ailleurs, de plus en plus facile grâce aux développements informatiques et aux nombreux outils disponibles pour les analyser efficacement (voir en particulier Diwersy et alii 2017). De la même manière, comme nous le décrirons infra (section 3.2), de nombreux besoins fondamentaux ou appliqués en diachronie sont renouvelés et financés (tels que la gestion de l'évolution des connaissances, les problématiques liées à la néologie, etc.).

dernier groupe d'obstacles, liés aux besoins de réflexion théorique, semble donc celui qui appelle aujourd'hui le plus de recherches. Dans ce contexte, nous proposons d'adopter le prisme de la terminologie textuelle afin d'alimenter cette réflexion. La terminologie textuelle est née du rapprochement entre les corpus de textes et la terminologie. Les textes spécialisés sont alors considérés comme des « réservoirs » de connaissance et l'intérêt de cette optique réside dans l'analyse des usages réels, en discours. Constituée donc pour pallier les inconvénients d'une vision trop prescriptive (Bourigault \& Slodzian 1999; Condamines 2005; Condamines \& Picton 2015), cette approche cherche depuis plusieurs années, d'une part, à systématiser les méthodes outillées d'analyse de la variation en corpus et, d'autre part, à théoriser différents aspects de l'analyse de la variation, à partir de contextes réels d'application. Bien qu'elle ne soit pas la seule à prendre en compte la diachronie, cette systématicité et la richesse des problématiques abordées en diachronie par cette approche participent ainsi à répondre aux quatre types d'obstacles identifiés. Ces travaux ont amené à questionner notamment plusieurs "piliers » d'analyse autour des notions de corpus, outils, experts et interprétation qui alimentent la réflexion théorique sur la diachronie (par ex. Picton 2014). À travers ce point de vue, nous souhaitons ainsi montrer la manière dont une approche outillée sur corpus contribue à lever les trois premières familles d'obstacles décrits et à nourrir la quatrième famille, théorique.

\section{Approche outillée en terminologie textuelle}

\subsection{Jalons méthodologiques}

11 L'approche de la terminologie textuelle repose sur ce que l'on pourrait appeler un «trépied méthodologique» (Picton \& Dury 2015), à savoir: des textes (corpus à comparer), des outils et des indices (dont les termes sont les points d'entrée pour l'analyse) et des entretiens avec les experts (afin de co-construire une interprétation des données). Cet ensemble est guidé pendant le processus par un besoin, le plus souvent appliqué, à l'origine de l'analyse.

Ce processus peut être divisé en plusieurs tâches, chacune impliquant différents protagonistes (Tableau, adapté de Condamines \& Picton 2015).

Tableau 1. Approche de la terminologie textuelle : tâches et protagonistes

\begin{tabular}{|l|l|}
\hline Tâches & Protagonistes \\
\hline
\end{tabular}




\begin{tabular}{|l|l|}
\hline $\begin{array}{l}\text { Identification d'un besoin } \\
\text { (éventuellement sa «traduction » en termes linguistiques) }\end{array}$ & $\begin{array}{l}\text { utilisateurs } \\
\text { experts du domaine } \\
\text { experts linguistes }\end{array}$ \\
\hline Compilation d'un corpus & $\begin{array}{l}\text { experts linguistes } \\
\text { experts du domaine }\end{array}$ \\
\hline Identification et mise en œuvre des outils pertinents & $\begin{array}{l}\text { experts linguistes } \\
\text { (éventuellement } \\
\text { informaticiens) }\end{array}$ \\
\hline Interprétation des indices fournis par les outils & experts linguistes \\
\hline Construction d'une interprétation finale & experts linguistes \\
& experts du domaine \\
\hline $\begin{array}{l}\text { Théorisation des résultats (apport linguistique } \\
\text { reproductibilité) }\end{array}$ & experts linguistes \\
\hline
\end{tabular}

Tout au long de ces étapes, plusieurs types d'outils sont mis en œuvre, tels que des extracteurs de termes, des analyseurs syntaxiques, des programmes ad hoc et des concordanciers. Ces outils permettent en effet d'observer différents types d'indices dans les corpus, appartenant à quatre familles ${ }^{2}$, illustrées à la section 3.3 :

- indices de types quantitatifs : calculs et comparaisons de fréquences, analyses statistiques sur l'absence ou la présence d'une unité ou d'un phénomène particulier dans les corpus ;

- observation des variations de forme (variantes lexicales, graphiques, orthographiques ou syntaxiques, avec l'hypothèse qu'une variation de forme peut révéler une variation conceptuelle [voir entre autres Cabré $1998: 241$ ou Tartier 2004 sur cette question]);

- analyse de la distribution des unités et de sa variation pour analyser des comportements sémantiques spécifiques (ressemblances et différences de sens entre unités). Cet indice peut être mis en œuvre de deux manières :

- sans interprétation a priori, c'est-à-dire un repérage de similarités ou divergences sémantiques sur la base de l'observation de la distribution des unités (syntaxique ou cooccurrence de $\mathrm{n}$ unités à gauche/droite, voir par exemple Condamines \& Rebeyrolle 1997; Habert 2005).

- avec interprétation a priori, c'est-à-dire l'observation de cooccurrents définis en amont de l'analyse et spécifiquement ciblés pour cette analyse afin de servir de «marqueurs » (Meyer 2001) de présence d'une information pertinente (cf. infra).

\subsection{Contextes d'application}

La terminologie textuelle s'est développée à l'origine autour de demandes appliquées faisant intervenir le besoin d'analyses linguistiques. Les premiers travaux dans cette approche ciblaient ainsi la construction de ressources termino-ontologiques à partir de textes (Aussenac-Gilles \& Condamines 2004). Avec le développement de cette thématique et le besoin de maintenance de ces ressources dans le temps (AussenacGilles et alii 2007), cette approche s'est très vite intéressée à la dimension diachronique et aux possibilités méthodologiques en corpus pour l'aborder. À ce jour, nous avons 
appliqué cette approche outillée dans trois types de contextes de recherche en diachronie, décrits dans les sections suivantes.

\subsubsection{Repérage de l'évolution en diachronie courte (contexte [a])}

Cette première demande a été formulée par le Centre national d'études spatiales (Cnes) et concernait le repérage de l'évolution des connaissances dans les projets spatiaux. Cette évolution entraîne en effet des difficultés non négligeables dans la pratique des experts du domaine, telles que : mauvaise communication entre ingénieurs, oubli du contexte de connaissances dans lequel le projet a été initié, plus généralement, modification non consciente du sens/de la forme des termes, etc. Cette question est particulièrement centrale dans le cadre de la mise en place de projets dits « de longue durée " (une dizaine à une trentaine d'années) pendant lesquels les connaissances convoquées évoluent nécessairement, parfois insidieusement. À travers la comparaison outillée du fonctionnement des termes dans des documents sur un même projet à différentes périodes, nous avons exploré trois problématiques : (a) dans quelle mesure les textes du domaine reflètent cette évolution, (b) quels sont les indices linguistiques mis en jeu dans les textes pour la marquer et (c) quels sont les moyens outillés pour les repérer (Picton 2009 ; 2014) ? Pour ce faire, nous avons travaillé à partir de deux corpus en diachronie courte: trois éditions successives d'un cours en optique et optoélectronique spatiale (1994, 1998 et 2002) et les cahiers des charges de la balise DORIS, première et troisième génération (1989 et 2000).

\subsubsection{Repérage et caractérisation de la déterminologisation (contexte [b])}

Cette deuxième demande a également été formulée par le Cnes qui, à l'occasion de son $50^{\mathrm{e}}$ anniversaire, souhaitait comprendre la manière dont le spatial, en tant qu'ensemble de connaissances, réelles ou imaginaires, se diffuse auprès du grand public ${ }^{3}$. À travers notre regard de linguiste, nous avons mis en lien cette demande avec la notion de déterminologisation ${ }^{4}$, c'est-à-dire l'analyse de l'intégration des termes dans la langue générale, en tant que processus et résultat. Pour ce faire, l'analyse a été fondée une fois encore sur une comparaison outillée du fonctionnement des termes, dans des corpus de différents genres et degrés de spécialité, supposés refléter différentes étapes de l'intégration des termes dans la langue générale (Humbert-Droz 2017): articles scientifiques et thèses, communiqués de presse et rapports de laboratoires, articles de vulgarisation et articles de presse. Ce phénomène se déroulant nécessairement dans le temps, ces corpus ont été organisés en sous-corpus comparables en diachronie courte, de 2003 à 2016.

\subsubsection{Analyse de convergences et divergences de terminologies entre groupes de locuteurs-experts (contexte [c])}

17 Cette troisième demande n'est pas une demande institutionnelle, mais est le fruit d'un besoin exprimé par des experts du domaine de la pédagogie universitaire. Dans ce domaine, les experts sont notamment des chercheurs, mais également des conseillers pédagogiques. Fréquemment, les mêmes personnes peuvent être à la fois chercheurs et conseillers. Ces deux pratiques, recherche et conseil, amènent les experts à ressentir une variation et une évolution de la terminologie du domaine propres à ces deux groupes, distinctement. Pour tenter de saisir et mettre en évidence ces phénomènes 
ressentis, nous avons proposé une comparaison outillée du fonctionnement des termes dans des corpus de chercheurs et conseillers, en diachronie (2004-2014), en Suisse, au Québec et en Belgique (Picton 2017 ; Picton \& Dury 2017). Ces corpus ont ensuite été organisés en différents sous-corpus à comparer en fonction de la variation à observer (diachronie, diastratie et diatopie).

\subsection{Exemples de mise en œuvre}

Sur la base de ces trois contextes appliqués, nous présentons brièvement dans cette section plusieurs cas d'analyses diachroniques mettant en œuvre les quatre familles d'indices distingués supra.

\subsubsection{Observation d'indices quantitatifs}

Le premier indice repose sur l'observation de la présence ou de l'absence statistiquement significative de termes entre sous-corpus. Ceci permet d'observer à la fois des groupes de termes au fonctionnement quantitatif comparable (appelés termes « chrono-homogènes » (Habert et alii 1997 ; Picton 2014) et l'évolution de termes isolés. La Figure 1, tirée de l'analyse du contexte (a), présente une liste de termes « chronohomogènes » ayant connu une hausse statistiquement significative ${ }^{5}$ dans les différentes éditions du manuel formant le corpus, entre 1994 et 2002 (Picton 2009). Après discussion avec les experts impliqués dans le projet, une thématique centrale a pu être identifiée dans cette liste, celle de l'intégration mécano-optique des miroirs (termes indiqués en gras, Figure 1). Cette croissance est liée au développement de nouvelles techniques d'intégration des miroirs (telles que des nouvelles colles, la diminution des parasites, etc.) à la fin des années 1990.

Figure . Exemple de thématique en croissance en optique spatiale

Thématique identifiée : technologie et intégration mécano-optique des miroirs

Termes liés : APS, arêtes, baffle, cœur, colle, couches, déformation, diffusion, domaine spectral, face, fibre, image, milieu, mode, MOS, optique, parasites, photo, pixel, plan, polissage, TDI, transfert des charges, transition, utilisation

La Figure 2 ci-dessous, tirée du contexte (b) (Condamines \& Picton 2012), présente quant à elle l'évolution de termes renvoyant à différentes tailles de satellites qui, depuis les années 1980, sont la cible de progrès de miniaturisation. On trouve en effet aujourd'hui des mini-satellites (de 100 à $1000 \mathrm{~kg}$ ), des microsatellites (de 10 à 100kg), des nano-satellites (de 1 à 10kg), des pico-satellites (moins de $1 \mathrm{~kg}$ ) et des femtosatellites (moins de 100g). Ces deux graphiques illustrent en parallèle l'évolution diachronique des fréquences de quatre de ces termes dans les communiqués de presse et la presse, entre 1998 et 2012. 
Figure . Évolution comparée de fréquences : nouveaux types de " satellite ", dans la presse (à droite) vs dans des communiqués de presse (à gauche)
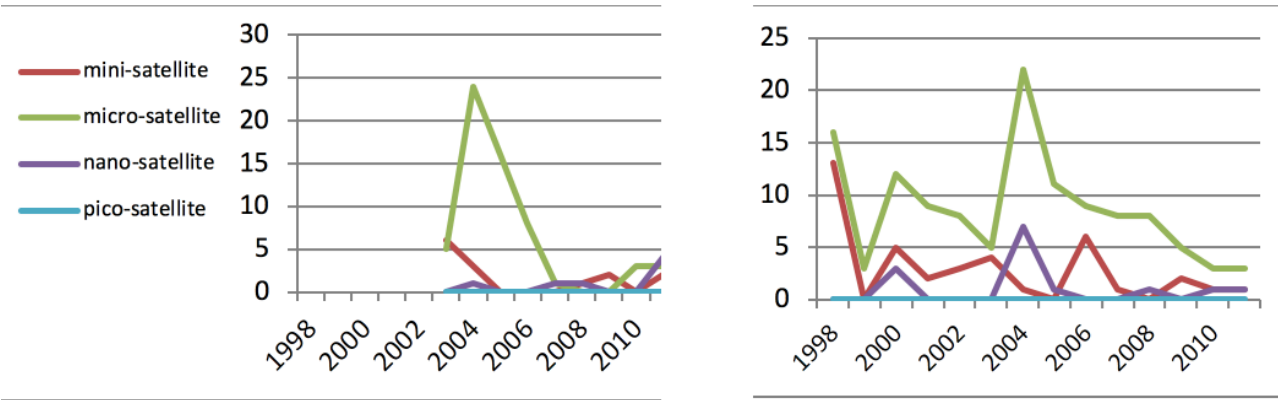

Ces courbes de fréquences permettent de visualiser d'une part le fait que tous ces termes sont attestés, tant dans les communiqués que dans la presse. D'autre part, le pic de croissance de 2004 (en vert), dû à une actualité forte dans le paysage spatial des microsatellites en $2004^{6}$, semble se répercuter immédiatement dans les deux souscorpus. Si l'on accepte le fait que les communiqués de presse servent de base à l'écriture des articles de presse, ces pics illustrent alors un lien de diffusion, au moins partiel, entre communiqués de presse et presse générale dans le temps.

\subsubsection{Variantes et répartition}

D'un point de vue diachronique, lorsqu'un terme ou un concept apparaît dans une langue de spécialité, plusieurs dénominations peuvent apparaître en concurrence, phénomène que Guilbert (1975) appelle «foisonnement néologique ». De ce point de vue, l'ensemble des variantes en concurrences dans une LSP peuvent être des indices de nouveauté. De la même manière, avec le temps, plusieurs de ces variantes tendent à diminuer ou disparaître et laissent l'avantage à une variante préférée, alors implantée (par ex. Quirion 2003), lorsque le concept ou le terme s'est stabilisé dans le domaine. Le Tableau 2 illustre ces phénomènes autour du concept de TELEVOTEUR, en pédagogie universitaire (contexte c). Ce concept apparaît au début des années 2000 sous la forme de trois dénominations (boitier de vote électronique, boîtier de vote électronique, boitier de vote), puis se développe et se stabilise après 2010 sous la dénomination télévoteur.

Tableau 2 - Variantes dénominatives du concept TELEVOTEUR et leur évolution en pédagogie universitaire

\begin{tabular}{|l|l|l|}
\hline Pédagogie universitaire & $\mathrm{D} 1$ (2004-2009) & $\mathrm{D} 2(2010-2014)$ \\
\hline télévoteur & 0 & $124(92.6 \%)$ \\
\hline boitier de vote électronique & $3(43 \%)$ & $1(0.7 \%)$ \\
\hline boitier de vote & $1(14 \%)$ & 0 \\
\hline boîtier de vote électronique & $3(43 \%)$ & $3(2.3 \%)$ \\
\hline boîtier de vote interactif & 0 & $1(0.7 \%)$ \\
\hline télécommande & 0 & $1(0.7 \%)$ \\
\hline
\end{tabular}




\begin{tabular}{|l|l|l|}
\hline système à réponses personnalisées & 0 & $2(1.5 \%)$ \\
\hline$S R P$ & 0 & $2(1.5 \%)$ \\
\hline
\end{tabular}

\subsubsection{Indices distributionnels sans interprétation a priori} changements sémantiques dans le temps. Ainsi, dans le Tableau 3, l'apparition de la dépendance syntaxique interface opérateur (en 2000, $3^{\mathrm{e}}$ génération de balises, contexte a) peut être liée à un changement de fonction de l'opérateur: autrefois en charge des opérations manuelles sur les instruments (tourner, pousser), ces actions ont été informatisées et s'opèrent à travers une interface.

Tableau 3 - Cas de opérateur dans le projet DORIS : changement de distribution

\begin{tabular}{|l|l|}
\hline $1^{\text {re }}$ génération de balises & $3^{\text {e }}$ génération de balises \\
\hline $\begin{array}{l}\text { appuie, tourne: actions «manuelles » de } \\
\text { l'opérateur }\end{array}$ & $\begin{array}{l}\text { interface : informatisation du travail de } \\
\text { l'opérateur }\end{array}$ \\
\hline
\end{tabular}

Un changement de dépendances peut également mettre en évidence l'apparition de nouveaux traits sémantiques pour un terme donné. Ainsi, dans le contexte (b), la large diffusion du terme navigation satellite dans la presse s'accompagne d'une augmentation de la couverture sémantique de ce terme, grâce au développement et à la démocratisation des technologies GPS. L'observation des modifieurs du terme navigation dans la presse montre l'apparition de l'adjectif terrestre, ajoutant ce trait sémantique à ce terme (auparavant uniquement lié aux concepts aérien, maritime ou internet), par exemple :

(i) « système de navigation des véhicules terrestres » (corpus presse)

(ii) «nous conjuguons les données GPS avec les données de la réflectométrie pour obtenir, notamment en matière de navigation terrestre, une précision jusque-là inégalée et aussi une plus grande fiabilité » (corpus presse)

\subsubsection{Indices distributionnels avec interprétation a priori}

Suivant le principe général des "contextes riches en connaissances" introduit par Meyer (2001), il est possible de repérer dans les textes des contextes porteurs d'une information pertinente pour un besoin terminologique donné. Ce type de contextes peut être repéré grâce à des marqueurs de relations conceptuelles (utiles en particulier pour la construction de réseaux terminologiques). Ainsi, une relation hyperonyme/ hyponyme pourra être mise en évidence par un marqueur tel que un $X[\operatorname{tel}(s)$ que / comme] $Y, Z, W$ :

(iii) une charge utile, telle que caméra, capteurs ou instrument de mesure (corpus spatial, contexte a)

Ces marqueurs, liés à l'objectif de l'étude, sont définis et pensés en amont de la recherche (donc a priori). Sur la base des trois contextes mentionnés supra, nous avons défini différents types de marqueurs, dont des marqueurs de la présence 
d'informations sur l'évolution d'un terme/concept/domaine, tels que ancien, prototype, nouveau, etc. Ainsi, ces marqueurs permettent d'identifier des contextes tels que (iv), riches en information sur l'évolution de concepts :

(iv) Signalons qu'actuellement de nouveaux produits, photodiodes avalanches à

très grandes surfaces et détecteurs hybrides entre PM et photodiode avalanche,

commencent à apparaître. (contexte a)

L'un des intérêts majeurs de cet indice est qu'il permet de travailler en synchronie dynamique (Guilbert 1973; Martinet 1990, section 4.3), point de vue qui permet de saisir des traces d'évolution en synchronie. Les contextes ainsi repérés indiquent pour la plupart une expression du sentiment linguistique (ou notionnel) des experts (par ex. Lecolle \& Achard-Bayle 2014). Ils permettent de repérer directement des jugements linguistiques des experts sur l'évolution de leurs termes, concepts et domaine, présents dans les textes.

\section{Quels questionnements et apports en diachronie?}

À travers ce panorama, nous avons décrit les méthodes développées par la terminologie textuelle pour l'analyse de la variation en corpus spécialisés, ainsi que plusieurs contextes appliqués d'analyses qui participent à la réconciliation entre diachronie et langues de spécialité. Afin de poursuivre notre réflexion, nous présentons dans cette section les principaux apports et questionnements soulevés par cette approche lorsqu'elle s'attache à l'analyse diachronique.

Bien que, dans les faits, ces aspects soient imbriqués, nous proposons d'organiser notre réflexion en trois points de vue : les apports et questionnements descriptifs, les apports et questionnements méthodologiques et les apports et questionnements théoriques, mis en avant par la terminologie textuelle.

\subsection{Apports et questionnement descriptifs}

30 Le premier point de vue proposé est celui du besoin d'enrichir les descriptions des fonctionnements des langues de spécialité et de leurs évolutions. Les LSP étant généralement définies comme relevant à la fois de la langue et des connaissances (par ex. Lerat 1995), il semble essentiel de bien poser la pluralité des niveaux de description pertinents.

31 Dans ce contexte, les principaux questionnements auxquels la terminologie textuelle tente de répondre à ce jour sont ceux :

- de la nature des phénomènes observables en diachronie courte dans une langue de spécialité 7 ,

- de la nature des phénomènes observables dans le processus de déterminologisation',

- des différences potentielles de nature des évolutions entre différents groupes (ou «strates ») de locuteurs-experts 9 .

Ces questionnements ont amené des éléments de réponses variés, associés aux différents contextes d'application mentionnés, et dont nous relevons ici ceux qui nous semblent les plus significatifs.

Premièrement, le repérage de l'évolution des connaissances dans les projets spatiaux de longue durée (contexte [a]) a permis de révéler la multiplicité des phénomènes en jeu 
sur de courtes périodes temporelles (à savoir 17 catégories d'évolutions, telles que la néologie de forme/de sens/complète, la néologie relative, la centralité, etc. $\left.{ }^{7}\right)$, à différents niveaux : au niveau de la dénomination, du concept, de l'instance, des genres, etc. De plus, si cette évolution peut souvent être mise en lien avec la néologie, elle peut également renvoyer à la notion de «centralité ». Cette notion est décrite par Jacques (2005 : 306) comme le « fait que, dans une sphère d'activités donnée, certains référents sont plus au centre des préoccupations des locuteurs de cette sphère d'activité que d'autres référents ", la diachronie permettant de préciser que cette préoccupation peut se dérouler pendant une période donnée. Ces résultats, obtenus sur de très courts intervalles temporels, participent de fait à la «légitimation » de la diachronie courte pour l'analyse des langues de spécialité. Si ce point de vue diachronique existe en langue générale (notamment à travers la notion de brachychronie [Mair \& Leech 2006] ou encore des travaux de Siouffi et alii 2012 par exemple), il prend une dimension tout à fait centrale pour l'évolution les langues de spécialité, qui, entre langue et connaissances, évoluent potentiellement très rapidement, au rythme des domaines auxquels elles sont liées.

Deuxièmement, le contexte (b), dans le champ de la déterminologisation, permet de développer des analyses de fonctionnements lexico-syntaxiques particuliers, présents en LSP. L'analyse outillée de corpus a ainsi permis de montrer en particulier une tendance forte à rétablir les prépositions dans les termes complexes, lorsqu'ils sont intégrés dans la langue générale (comme le terme données satellite et sa variante données du satellite, plus fréquente en langue générale [Condamines \& Picton 2012]). D'autres phénomènes, tels que la multiplication des points de vue accompagnant la néologie sémantique, sont actuellement en cours d'observation (Humbert-Droz en cours; Condamines et alii 2018), résultats qui permettent de reproblématiser la question de la néologie dans une perspective diachronique.

Enfin, le contexte (c), qui s'appuie sur une analyse contrastive de l'évolution de la langue de spécialité de la pédagogie universitaire, a permis de mettre en évidence les temporalités et évolutions différentes dans différents groupes d'experts d'un même domaine. Ainsi, pour reprendre l'exemple de l'implantation du terme télévoteur (section 3.3.2), la comparaison des évolutions d'usage entre conseillers et chercheurs dans ce domaine montre deux choix terminologiques différents dans le temps: alors que les chercheurs utilisent plutôt le terme télévoteur, les conseillers lui préfèrent zappette, dans nos corpus. Ce court exemple met en particulier en évidence le fait que la diachronie doit amener à réfléchir à la diastratie et à l'existence de différents groupes d'experts au sein d'un même domaine, aux évolutions et à leurs intérêts propres.

\subsection{Besoins et questionnement méthodologiques}

Nous l'avons souligné au début de cet article, les approches méthodologiques en diachronie en LSP présentent à ce jour un profil assez hétérogène et riche. Pourtant, dans un contexte scientifique, il semble de première importance de chercher à accompagner les descriptions d'un cadre méthodologique transparent et reproductible.

Dans ce contexte, l'un des apports méthodologiques majeurs de la terminologie textuelle repose sur la proposition d'un cadre méthodologique (certes perfectible) reproductible et systématique d'analyse en corpus, basé sur quatre indices (section 3.3). Cette proposition permet d'articuler approches quantitatives et qualitatives et de 
travailler à partir de «faisceaux » d'indices pour construire une interprétation fine des données.

Suivant ce cadre méthodologique, la terminologie textuelle a surtout contribué à répondre à deux types de questionnements. Le premier est celui de mesurer la possibilité d'exploiter l'hypothèse d'un parallèle entre langue et connaissances pour repérer l'évolution en corpus (Cabré 1998) ; autrement dit, la possibilité d'interpréter la présence d'une évolution de connaissances sur la base du repérage d'une évolution formelle (présence de variantes par exemple). Le second questionnement concerne précisément l'équilibre nécessaire entre les phénomènes repérés en corpus, l'implication et les connaissances des experts, ainsi que les connaissances de l'analyste pour parvenir à un consensus et à une interprétation pertinente des données.

Les réponses de cette approche à ces deux questionnements se déclinent comme suit: du point de vue du parallèle possible entre langue et connaissances, cette approche méthodologique, reproduite dans plusieurs contextes variés, amène à confirmer le fait que cette hypothèse est exploitable: une évolution formelle repérable en corpus diachronique peut révéler la présence d'une évolution de connaissances. Néanmoins, suivant également les conclusions de Tartier (2004), il ne semble pas possible de lier une interprétation unique et directe à un phénomène formel identifiable. Ce lien se construit, ou plutôt se "co-construit ", lors de l'analyse à travers des discussions avec les experts et la prise en compte des besoins qui ont suscité l'analyse. De fait, cette situation amène à établir un équilibre entre corpus, outils, linguistes et experts de domaine. Ce «partenariat» (pour reprendre les termes de Bourigault \& Slodzian 1999), en diachronie, est confronté cependant à une difficulté spécifique: celle de la complexité à poser un « rétrodiagnostic » (voir Dury 2011 ; Dury 2018), c'est-à-dire à la capacité des experts à poser un jugement sur leurs usages passés. Du point de vue de l'approche outillée présentée ici, une proposition consiste à prendre en compte le sentiment linguistique des experts dans les corpus, à travers le repérage d'éléments métalinguistiques et de marqueurs de leur sentiment linguistique (indice distributionnel avec interprétation a priori). Cette proposition, travaillée conjointement avec Pascaline Dury en particulier (Picton \& Dury 2015), permet de mettre en évidence le fait que la place de l'expert dans l'analyse ne prend pas seulement la forme d'entretiens oraux ou écrits : les avis et propositions des experts, à travers l'expression de leur sentiment linguistique, sont très présents dans les textes et le linguiste peut/ doit en tenir compte dans son analyse.

\subsection{Besoins et questionnement théoriques}

40 Les différentes études présentées dans cet article se révèlent être un lieu privilégié de réflexion théorique sur la diachronie et les langues de spécialité. Si les nouvelles théories de la terminologie font, de manière générale, la part belle à la diachronie et à la variation, la terminologie textuelle semble celle qui a le plus approfondi cette question et à en avoir systématisé l'étude, à partir de problématiques concrètes et variées. Comme nous l'avons ébauché tout au long de cet article, les éléments théoriques discutés par la terminologie textuelle concernent aussi bien la légitimation de l'étude de la diachronie en langues de spécialité que l'exploration des liens entre néologie et diachronie ou encore la place et le rôle de l'expert pour l'analyse. 
41 Elle participe ainsi en général à théoriser la question de la diachronie en langues de spécialité et à en légitimer la place, en particulier en mettant en avant la rapidité des changements conceptuels et formels touchants les langues de spécialité. Elle éclaire ainsi l'importance de la diachronie courte.

Les besoins et difficultés spécifiques de l'analyse diachronique en corpus ont également mené à une prise de conscience quant au manque de réflexion sur la question du rôle et de la place de l'expert. Si cette question est centrale en langues de spécialité de manière générale, elle est rarement discutée. Cependant, la collaboration avec les experts en diachronie la rend incontournable et appelle à une réflexion de fond, amorcée dans ces recherches à travers la notion de "co-construction » de l'interprétation et la prise en compte du sentiment linguistique des experts dans les textes, à travers la notion de métalangage.

Mais, malgré ces contributions d'ordre théorique, les besoins de réflexion sur la diachronie et les LSP restent nombreux, afin d'en consolider la place. Au moins deux grands types de besoins peuvent être identifiés. Le premier concerne la mise en évidence des spécificités de la diachronie, lorsqu'elle est appliquée aux langues de spécialité : peut-on en distinguer et, si oui, lesquelles ? Le second axe concerne le statut de la variation diachronique, par rapport aux autres types de variations dialogiques existants en langues de spécialité. Les paragraphes suivants cherchent à lancer et alimenter la réflexion sur ces deux axes.

Du point de vue des spécificités de la diachronie, lorsqu'elle concerne les langues de spécialité, nous avons déjà mis en avant par exemple la place particulière de la diachronie courte (section 4.1), mais également l'importance de prendre en compte le lien entre langue et connaissances pour saisir la richesse de l'évolution propre des langues de spécialité. Ce dernier point pourrait se refléter dans les choix terminologiques désignant les types d'analyse diachronique choisis par les études en langues de spécialité, et qui méritent d'être affinés.

À titre de comparaison, en langue générale, Marchello-Nizia 1995 propose une distinction entre :

- la linguistique diachronique, où l'on fait abstraction de la dimension externe (c'est-à-dire un ensemble de phénomènes extralinguistiques) pour se concentrer sur l'évolution interne du système linguistique (c'est-à-dire les changements lexicaux, syntaxiques, sémantiques, etc.) ;

- et la linguistique historique, où l'on associe perspectives interne sur le système linguistique et externe pour retracer l'évolution de la langue: ces recherches étudient l'évolution interne du système et l'expliquent, en partie du moins, par l'histoire externe de ce même système.

L'on peut ajouter un troisième élément : la notion d'histoire de la langue (par ex. Antoine 1981 ; Brunot 1967 ; Helgorsky 1981), perspective qui ne s'intéresse qu'à la dimension externe de l'histoire d'une langue, "ses succès et ses revers", pour reprendre les termes de Brunot (ibid.).

En terminologie, Pascaline Dury (2018), en insistant sur la centralité de la diachronie courte pour la terminologie propose de distinguer, quant à elle, linguistique historique et linguistique diachronique, sur la base d'une distinction présente dans quelques ouvrages de linguistique diachronique tels que celui de Joseph \& Janda (2003). L'auteure explique alors 
que l'on [peut] faire de la linguistique historique sans qu'il s'agisse pour autant de linguistique diachronique: la linguistique historique consistant à observer des périodes temporelles anciennes, voire très anciennes sans forcément s'attacher à décrire les mouvements d'une langue et la linguistique diachronique relevant ce qui a changé (ou pas) dans une langue au fil d'un temps qui peut être proche ou très proche. (Dury $2018: 31$ ) distinction entre une analyse qui vise à décrire la dynamique des évolutions (et qui est alors appelée très justement diachronique, "à travers le temps ») et une analyse plus "statique» (voire synchronique), dite «historique»; deuxièmement, puisqu'elle définit la linguistique diachronique comme étant plutôt portée sur des analyses des évolutions temporellement "proches ou très proches ", elle fait la part belle à une diachronie courte, contemporaine. Cependant, il nous semble que cette distinction, lorsqu'elle s'applique aux langues de spécialité, n'est pas tout à fait satisfaisante. Tout d'abord, elle ne permet que difficilement d'envisager des cas d'analyses diachroniques (c'est-à-dire du mouvement d'une langue dans le temps), courte, sur des périodes anciennes, pourtant possibles et pertinentes pour les LSP. De plus, la notion d'« ancienneté » peut d'être difficile à trancher : parle-t-on d'états de langue tels que le Moyen Français, ou un siècle suffit, surtout pour certaines langues de spécialité assez «jeunes» (par exemple celle de l'informatique) ? Enfin, ce point de vue ne permet pas de souligner la centralité des deux dimensions prises en compte dans l'analyse des langues de spécialité, à savoir la langue (interne) et les connaissances (externes).

Pour répondre à ces limites, une autre manière de conceptualiser les choses, un peu plus proche des propositions de Marchello-Nizia, serait une distinction reposant plus sur l'idée de dimensions interne et externe que sur la période temporelle considérée par les analyses. Ainsi, l'on pourrait distinguer pour les langues de spécialité :

- d'une part une approche diachronique, qui s'attache à l'étude de l'évolution d'une LSP donnée, que celle-ci soit considérée en diachronie courte ou longue, sur des époques contemporaines ou temporellement éloignées. Étant donnée la nature des LSP, cette approche peut ensuite se décliner en trois points de vue nuancés :

- une approche diachronique plutôt centrée sur la langue (dimension interne)

- une approche diachronique plutôt centrée sur les connaissances (relevant de la dimension externe)

- une approche diachronique centrée sur le lien entre langue et connaissances (à l'instar de ce que nous avons présenté dans cet article);

- d'autre part une approche historique, qui focalise son attention sur les éléments externes (tels que politiques, scientifiques et culturels) susceptibles d'expliquer l'évolution des LSP (tant du point de vue de la langue que des connaissances). Une fois encore, cette approche pourrait être qualifiée de «courte» ou «longue» en fonction de l'intervalle temporel considéré.

50 À cette distinction, nous pouvons encore ajouter deux autres points de vue, complémentaires, pour étudier l'évolution des langues de spécialité.

51 Le premier est celui de l'approche en synchronie dynamique. Cette notion, introduite originellement par le courant fonctionnaliste (entre autres Martinet, 1990 ; Houdebine 1985 : 7) repose sur l'idée de « la coexistence éventuelle d'usages différents d'une entité dans le discours, à un moment donné » (Feuillard 2007: 5). Ceci représente une perspective très pertinente, en particulier pour la terminologie et la néologie, « dans la mesure où la synchronie qu'elle étudie est toujours prise dans le mouvement qui est le 
'lieu de rencontre entre l'innovation et l'archaïsme' (Guilbert 1973)» (cité dans Gaudin $2003: 163)$.

Enfin, le second point de vue est celui de l'histoire des sciences. La différence qui s'opère entre approche historique et histoire des sciences s'appuie sur leur objet d'étude : l'approche historique travaille sur une LSP et peut chercher à mettre en avant l'évolution de celle-ci par des éléments historiques connus par discipline donnée; l'histoire des sciences quant à elle ne relève pas des langues de spécialité et cherche moins directement à établir de liens ente l'évolution d'une discipline et l'évolution de « sa » LSP. Il s'agit donc plutôt d'une complémentarité ${ }^{10}$.

Pour revenir à la seconde question posée supra (quel est le statut de la diachronie, comme variation, par rapport aux autres types de variations dialectales?), la prise en compte de la diachronie ouvre, selon nous une réflexion très riche quant à la place de cette variation parmi les autres types de variations dialectales (Freixa 2006). Premièrement, une revue de la littérature dans le domaine des LSP indique clairement la position privilégiée de la diachronie : celle-ci se révèle être, finalement, bien plus souvent décrite que les autres types de variations dialogiques, et notamment diastratique (selon les groupes/strates de locuteurs) et diatopique (géographique).

Deuxièmement, les différents contextes de recherche explorés dans nos études nous amènent à postuler le besoin de prendre en compte la variation diachronique en lien étroit avec l'analyse d'autres types de variations. Ainsi, par exemple, dans le cadre de la déterminologisation, le processus de passage des termes d'une LSP à la langue générale (entraînant donc un changement de groupe de locuteurs) se produit nécessairement dans le temps. La diachronie est donc, pour ainsi dire, sous-jacente à la variation diastratique dans ce cas. De plus, la comparaison des usages de différents groupes d'experts en pédagogie universitaire a permis de mettre en évidence le fait que les langues de spécialité sont touchées par différents types de variations, simultanément, dont certaines se révèlent dans le temps, en diachronie (Picton \& Dury 2017). Ainsi, une proposition serait de réfléchir à explorer les langues de spécialité comme de véritables diasystèmes (Coseriu 1998), notion empruntée à la linguistique variationnelle et renvoyant à une conception dynamique des langues comme des systèmes dont l'architecture se compose de différentes variétés linguistiques liées aux dimensions temporelles, géographiques et sociales (Dostie \& Hadermann 2016 ; Verjans 2013).

Cette proposition de prise en compte dynamique de différents types de variation doit également être mise en lien avec de nouveaux besoins et de nouveaux défis de l'analyse diachronique des langues de spécialité aujourd'hui. Nous en donnons quelques exemples dans la section suivante, toujours à travers le prisme d'une approche outillée en terminologie et de la définition de nouveaux besoins appliqués.

\section{Perspectives de la diachronie et nouveaux défis}

\subsection{Exemples de nouveaux besoins/demandes}

Actuellement, notre équipe travaille sur deux axes de recherche, qui amènent à développer notre réflexion sur la diachronie dans les langues de spécialité, en lien avec la diatopie et la diastratie. 
Le premier concerne la continuité des recherches présentées supra sur la déterminologisation. En particulier, dans sa thèse (en cours), Julie Humbert-Droz propose de développer un cadre méthodologique pour caractériser le phénomène de déterminologisation, non pas comme résultat seul d'une intégration de termes dans la langue générale, mais comme processus d'intégration, dans le temps. Elle propose dans ses recherches la construction d'un corpus plus équilibré entre types de variations (diachronique et diastratique) en physique des particules. Ce corpus se compose de textes de 2003 à 2016, répartis entre articles scientifiques et thèses, communiqués de presse et rapports de laboratoire, articles de vulgarisation et articles de presse (Humbert-Droz 2017), représentant différents groupes de locuteurs par lesquels ces termes sont utilisés.

Le second contexte est celui de la constitution de la Humanitarian Encyclopaedia ${ }^{11}$. Cette " encyclopédie ", à l'initiative du CERAH ${ }^{12}$ et d'experts chercheurs et praticiens internationaux du secteur humanitaire, se veut être une ressource de référence descriptive synthétisant les différences et convergences d'usages selon plusieurs perspectives. En effet, bien que les experts semblent s'accorder sur des principes et valeurs communes, beaucoup de termes et concepts montrent des divergences et nuances importantes selon le temps (diachronie), l'origine géographique des acteurs de l'humanitaire (diatopie), leur organisation, mais aussi leur discipline d'origine (telles que la santé publique, le droit, etc.) (diastratie) (par exemple Collinson \& Elhawary 2012). Il est nécessaire de chercher à repérer et décrire ces usages, dans toute leur finesse et dans toute leur variation. Un corpus comparable et représentatif de plusieurs de ces dimensions est en cours de constitution dans notre équipe.

\subsection{Différents défis et différents besoins}

Ces nouveaux contextes, de par la pluralité des types de variation à explorer, imposent d'affronter différents types de défis.

Du point de vue descriptif, et en lien avec ce que nous avons décrit supra, il s'agit de proposer des descriptions qui prennent en compte simultanément plusieurs types de variations (la diachronie, la diastratie et la diatopie) et qui parviennent à mettre en évidence leur(s) lien(s) éventuels.

Du point de méthodologique, ce contexte impose de développer des méthodes qui permettent d'explorer ces dimensions simultanément, autant que possible. Ceci amène à réfléchir en particulier à la construction de corpus «multi-comparables » adéquats, ainsi qu'aux outils qui permettent de manipuler et d'observer efficacement des données complexes et lourdes.

62 Enfin, d'un point de vue théorique, ces contextes s'inscrivent dans la continuité des réflexions amorcées et présentées dans cet article, en développant l'étude des différents types de variation en LSP et en travaillant la pertinence de considérer les LSP en tant que « diasystèmes » (supra).

Afin d'illustrer les besoins créés par ces nouveaux défis diachroniques et pour ouvrir notre propos, nous présentons trois axes de réflexion quant aux besoins méthodologiques liés à ces nouveaux contextes. Il s'agit là de recherches en cours et nous nous limitons ici à présenter ces besoins et des pistes de réponses encore élémentaires ${ }^{13}$. 

variation, dont la variation diachronique :

1. la question du découpage temporel des données à comparer ;

2. la question de la visualisation de données complexes à manipuler ;

3. la question de la modélisation des phénomènes, afin de faire émerger des comportements difficilement décelables à « l'œil nu » dans ce type de données.

65

(a) de deux stratégies: les sous-corpus sont découpés et organisés soit en fonction d'un événement extralinguistique (dans notre exemple de la physique des particules, il pourrait s'agit de la découverte du Boson de Higgs), soit sur la base d'un découpage régulier (par exemple tous les 5 ans). Ces stratégies présentent cependant plusieurs limites: premièrement, elles impliquent une certaine arbitrarité dans les choix de découpage, qui peut générer du silence dans les résultats obtenus, si une évolution ne correspond pas au découpage choisi. En particulier, dans le premier cas, si l'un des objectifs de l'analyse est de chercher à saisir les liens entre dimension temporelle et changement de «strates » de locuteurs, l'intégration de certains termes auprès d'une nouvelle strate de locuteurs pourrait ne pas concorder avec le moment prédéfini arbitrairement en diachronie. Dans le second cas, un découpage en sous-corpus trop nombreux entraîne des limites techniques, ergonomiques et cognitives pour la gestion et l'observation de ces données. En réponse à cette situation, une proposition méthodologique explorée par la linguistique diachronique semble applicable aux LSP. Cette approche, endogène, consiste à étudier la variabilité du point de vue des documents afin de faire émerger de ces derniers des regroupements. À travers une classification ascendante hiérarchique par contiguïtés (variability-based neighbor clustering, VNC), Gries \& Hilpert $(2008,2012)$ par exemple se fondent sur l'analyse du voisinage distributionnel des unités dans des corpus annualisés pour identifier des regroupements de documents possédant des propriétés communes et dégager ainsi des périodes pendant lesquelles les distributions indiquent une certaine homogénéité de fonctionnement. Les regroupements ainsi créés permettent alors d'organiser automatiquement les sous-corpus en périodes temporelles à analyser, minimisant ainsi la subjectivité dans le découpage.

Du point de vue de la visualisation, l'objectif est avant tout de soutenir les analyses en limitant l'impact de la lourdeur des données à manipuler et en facilitant la mise en évidence de phénomènes pertinents difficilement identifiables "à l'œil nu ». Pour la diachronie, une piste à explorer, et une fois encore présentée par les linguistes diachroniciens (dont Hilpert 2011) est celle des MotionCharts (Gesmann \& De Castillo 2011). Ces Motioncharts sont des graphiques dynamiques qui permettent d'observer plusieurs dimensions simultanément, dont l'une est la dimension temporelle. Par exemple, dans le cas de l'évolution d'une unité ou d'un concept et de son passage d'un groupe de locuteurs à un autre, ces graphiques dynamiques permettent de modéliser les fréquences d'utilisation entre ces deux groupes de locuteurs et de "dérouler " visuellement, sous forme de vidéo, les mouvements de fréquences d'un terme ou d'un groupe de terme dans la période souhaitée. La Figure 3 illustre ce type de graphique, pour le terme Boson de Higgs, et ses variantes principales en physique des particules. Les fréquences dans la presse sont en abscisse et celles dans les corpus spécialisés en ordonnée. La "frise» chronologique selon laquelle est déroulé le visionnage est indiquée en bas (ici figée à l'année 2012). 
Figure . MotionChart, évolution comparée de boson de Higgs et de ses variantes dans la presse et dans les communiqués

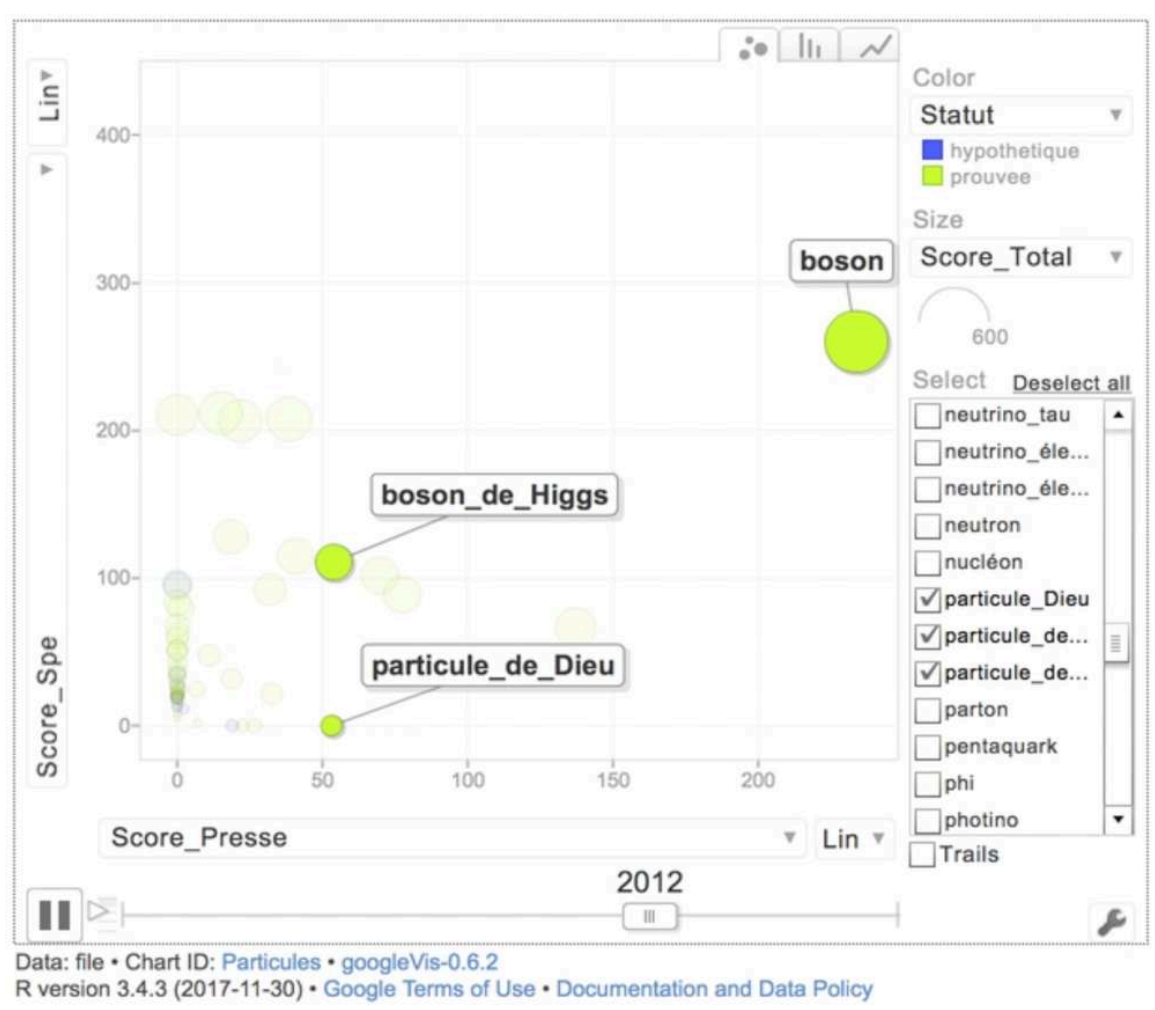

67 Enfin, toujours dans l'objectif de faire émerger de données complexes (appelées ici «multi-comparables») des phénomènes pertinents, l'analyse distributionnelle peut être exploitée pour observer des regroupements lexicaux et s'intéresser aux phénomènes qui touchent directement les termes. Les travaux récents dans cette optique se fondent essentiellement sur l'exploitation des plongements de mots ou plongements lexicaux (word embeddings) qui ont pour but de capturer le sens en contexte des unités lexicales en les représentant sous forme de vecteurs numériques (Mikolov et alii 2013 ; Ferré 2017). L'objectif de ces approches est de chercher à générer des "graphes" qui regroupent les unités lexicales associables à des sens donnés, émergeant dans le temps, et/ou en lien avec d'autres types de variation. Ces graphes offrent également une visualisation de ces regroupements sémantiques. La Figure 4 en offre un exemple en diachronie, sur les 100 ans du journal Le Devoir, autour de l'adjectif « gai » et son évolution sémantique entre 1900 et 2000. 
Figure . Graphe : regroupements sémantiques et changements sémantiques de l'adjectif gai (100 ans du Devoir, Picton \& Drouin 2017)

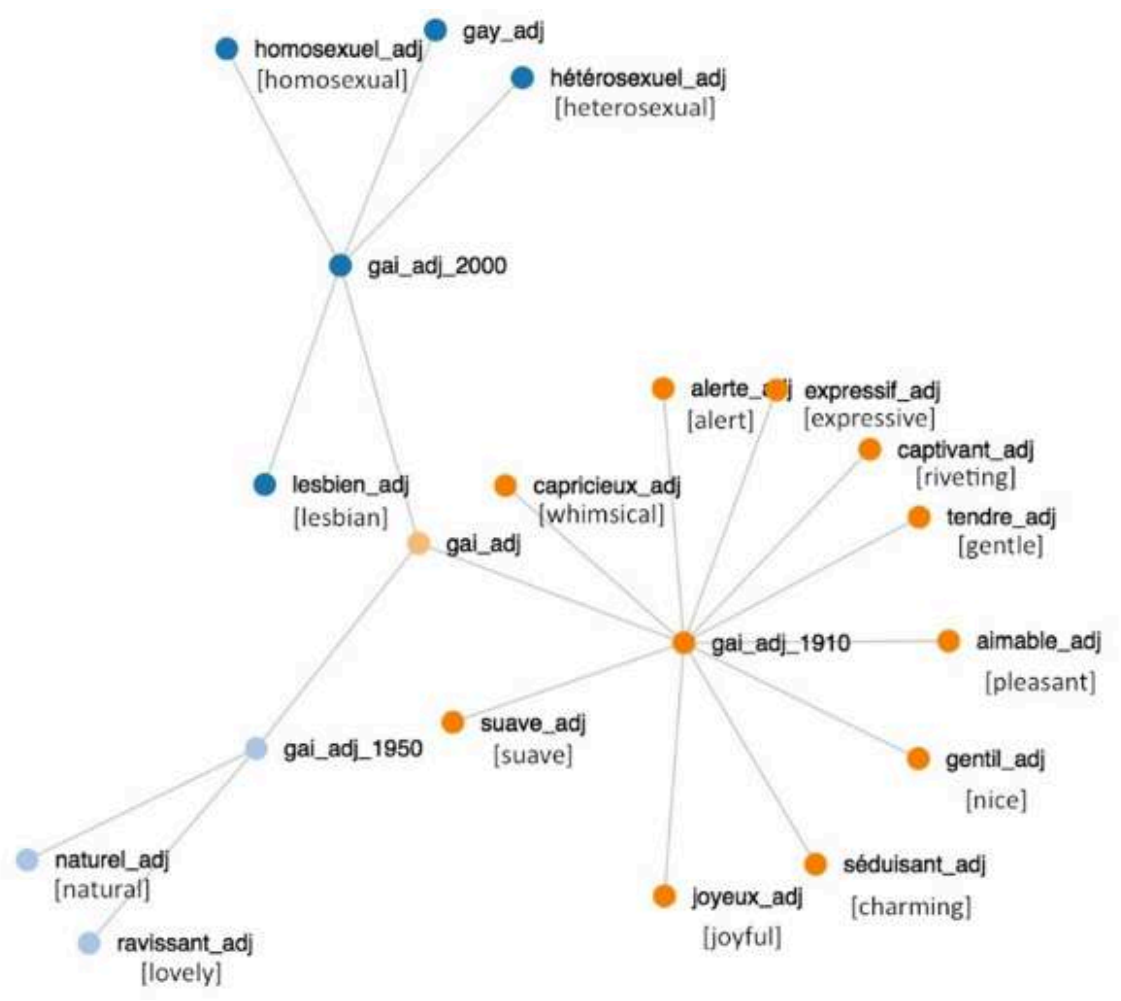

\section{Remarques conclusives}

Dans cet article, à travers le prisme de la terminologie textuelle, nous avons souhaité mettre en évidence le très grand chemin parcouru par les langues de spécialité dans leur réconciliation avec la diachronie. Cette dynamique est accompagnée d'une richesse et d'une pluralité d'études et recherches remarquables. Néanmoins, le besoin d'approfondissement de la réflexion théorique sur la question de la diachronie en langues de spécialité se fait clairement ressentir aujourd'hui, besoin nourri par le renouvellement des problématiques associées à la diachronie, par l'accès à de nouvelles demandes, à de nouveaux outils et à de nouveaux défis. Ces nouvelles ouvertures laissent toutefois présager un bel élan pour fonder cette réflexion. La voie est maintenant ouverte et le chemin tracé pour décrire et comprendre les langues de spécialité dans toute leur finesse, dans toute leur variabilité et stabiliser ainsi la place de la diachronie en langues de spécialité.

Nous tenons à remercier ici les personnes qui ont participé de près ou de loin aux différentes recherches diachroniques mentionnées dans cet article, et sans qui cette " réflexion diachronique " n'aurait pu voir le jour. Une mention toute particulière à Anne Condamines, Patrick Drouin, Pascaline Dury et Julie Humbert-Droz. 


\section{BIBLIOGRAPHIE}

ANToINE, G. 1981. «L'histoire de la langue. Problèmes et méthodes ». Le Français Moderne 49/2, 145160.

AUGER, P. 1973. Le vocabulaire forestier au Québec, des origines à nos jours. Étude historique. Thèse de doctorat en linguistique, Université de Strasbourg.

AUSSENAC-GILLES, N. \& A. CONDAMINES. 2004. « Documents électroniques et constitution de ressources terminologiques ou ontologiques ». Information - Interaction - Intelligence (I3) 4/1, 75-92. AUSSENAC-GILLES, N., A. CONDAMINES \& F. SÉDÈS (dir.). 2007. Évolution et maintenance des ressources termino-ontologiques. Information - Interaction - Intelligence (I3) Hors-série.

BANKS, D. 2012. « Diachronic ESP: at the interface of linguistics and cultural studies ». ASp [En ligne] 61, mis en ligne le 07 octobre 2014. DOI : 10.4000/asp.2738.

BOURIGAUlt, D. \& M. SLODZIAN. 1999. « Pour une terminologie textuelle ». Terminologies Nouvelles 19, 29-32.

BRUNOT, F. 1967. Histoire de la langue française des origines à nos jours. Paris : Armand Colin.

CABRÉ, M. T. 1998. La terminologie : théories, méthodes et applications. Ottawa : Armand Colin, Presses de l'Université d'Ottawa.

CANDEL, D. 2004. «Wüster par lui-même ». In CORTÈs, C. (dir.), Des fondements théoriques de la terminologie. Cahiers du C.I.E.L. (Centre Interlangue d'Études en Lexicologie), Université Paris 7, 1531.

CANDEL, D. \& F. GAUDIN (dir.). 2006. Aspects diachroniques du vocabulaire. Mont-Saint-Aignan : Publications des universités de Rouen et du Havre.

CHARPY, J.-P. 2011. « Les premiers dictionnaires médicaux en langue anglaise : glissements diachroniques du spécialisé au non spécialisé ». ASp [En ligne] 59, mis en ligne le 01 mars 2014. DOI : 2010.4000/asp.2176.

COLLINSON, S. \& S. ELHAWARY. 2012. « Humanitarian space: a review of trends and issues ». Overseas Development Institute (ODI), 32.

CONDAMINES, A. 2005. « Linguistique de corpus et terminologie ». Langages 157, 36-47.

CONDAMINES, A. \& N. DEHAUT. 2011. « Mise en œuvre des méthodes de la linguistique de corpus pour étudier les termes en situation d'innovation disciplinaire : le cas de l'exobiologie ». Meta :journal des traducteurs 56/2, 266-283.

CONDAMINES, A., J. HUMBERT-DROZ \& A. PICTON. 2018. « La diversification des points de vue comme processus de déterminologisation : étude en corpus dans le domaine de la physique des particules ». In Actes de la conférence Cineo 2018, Lyon (France).

CONDAMINES, A. \& A. PICTON. 2012. « Des communiqués de presse du Cnes à la presse généraliste. Vers un observatoire de la diffusion des termes ». In Actes de la conférence Langues de spécialité et néologie, Journées du CRTT (Centre de Recherche en Terminologie et Traduction), Lyon (France).

CONDAMINES, A. \& A. PICTON. 2014. «Étude du fonctionnement des nominalisations déverbales dans un contexte de déspécialisation ». In Actes du Congrès Mondial de Linguistique Française, Berlin (Allemagne). 
CONDAMINES, A. \& A. PICTON. 2015. «Terminologie outillée : analyse de corpus spécialisés dans différentes situations de néologie ». In Actes de la conférence conférence-hommage à John Humbley, "Quo vadis, Terminologia », Paris, France.

CONDAMINES, A. \& J. REBEYRolle. 1997. «Point de vue en langue spécialisée ». Meta, Presses de l'Université de Montréal XLII/1, 174-184.

COSERIU, E. 1998. « Le double problème des unités "DIA-S" ». Les cahiers DIA/Études sur la diachronie et la variation linguistique. Communication et Cognition, coll., 9-16.

DESMET, I. 2007. « Terminologie, culture et société. Éléments pour une théorie variationniste de la terminologie et des langues de spécialité ». Cahiers du Rifal, consulté le 15 avril 2018. <http:// termisti.ulb.ac.be/archive/rifal/PDF/rifal26/crf-26-00.pdf>.

DIWERSY, S., A. FALAISE, M.-H. LAY \& G. SOUVAY. 2017. « Ressources et méthodes pour l'analyse diachronique ». Langages 206/2, 21-44.

DOSTIE, G. \& P. HADERMANN (dir.). 2016. Diasystème et variation en français actuel : aspects sémantiques Carnets de lecture 29.

DROUIN, P., A. PICTON \& J. HUMBERT-DROZ. À paraître 2018. « Analyse des corpus spécialisés “multicomparables" à l'aide d'une approche outillée : quelles limites et quelles pistes de travail?». In Actes de la conférence LTT, Grenoble (France).

DURY, P. 1997. Étude comparative et diachronique de l'évolution. Thèse de doctorat en Lexicologie et Terminologie multilingues - Traduction, Université Louis-Lumière.

DURY, P. 2011. «Le sentiment d'un « besoin néologique » chez les experts pour remplacer des termes à connotation péjorative : quelques exemples tirés du domaine médical ». In Actes de la conférence Néologie et traduction spécialisée, $4^{\mathrm{e}}$ séminaire conjoint CVC-Termisti, Bruxelles, Belgique, 29 avril 2011.

DURY, P. 2013. «Que montre l'étude de la variation d'une terminologie dans le temps. Quelques pistes de réflexion appliquées au domaine médical ». Debate terminológico 9, 2-10.

DURY, P. 2018. « La dimension diachronique en anglais de spécialité : une approche terminologique ». Note de synthèse pour l'Habilitation à Diriger des Recherches : Université Paris 7.

DURY, P. \& A. Picton. 2009. « Terminologie et diachronie : vers une réconciliation théorique et méthodologique?». Revue Française de Linguistique Appliquée 13/2, 31-41.

FERRÉ, A. 2017. « Représentation de termes complexes dans un espace vectoriel relié à une ontologie pour une tâche de catégorisation ». In Actes de la conférence Rencontres des Jeunes Chercheurs en Intelligence Artificielle (RJCIA 2017), Caen (France).

FEUILLARD, C. 2007. « Grammaticalisation et synchronie dynamique ». La linguistique 43/1, 3-28.

FREIXA, J. 2006. « Causes of denominative variation in terminology - A typology proposal ». Terminology 12/1, 51-77.

GALISSON, R. 1978. Recherches de lexicologie descriptive : la banalisation lexicale. Paris : Nathan.

GAUDIN, F. 1993. Pour une socioterminologie - Des problèmes sémantiques aux pratiques institutionnelles. Rouen : Publications de l'Université de Rouen.

GAUDIN, F. 2003. Socioterminologie - Une approche sociolinguistique de la terminologie. Bruxelles : De Boeck - Duculot. 
GESMANN, M. \& D. DE CASTILLO. 2011. «Interface between R and the Google visualisation API. GoogleVis package for R. ", consulté le 2 juillet 2018 <http://cran.r-project.org/web/packages/ googleVis/googleVis.pdf>.

GRIES, S. T. \& M. HILPERT. 2008. « The identification of stages in diachronic data: variability-based neighbour clustering ». Corpora 3/1, 59-81.

GRIES, S. T. \& M. HILPERT. 2012. « Variability-based neighbor clustering: a bottom-up approach to periodization in historical linguistics ». In NEVALAINEN, T. \& E. TRAUGOTt (dir.), The Oxford Handbook of the History of English. Oxford : Oxford University Press, 134-144.

GUESPIN, L. 1995. « La circulation terminologique et les rapports entre science, technique et production ». Meta, Presses de l'Université de Montréal LX/2, 206-215.

GUILBERT, L. 1973. «Théorie du néologisme ». Cahier de l'Association Internationale des Études Françaises 25, 9-29.

GUILBERT, L. 1975. La créativité lexicale. Paris : Larousse Université.

HABERT, B. 2005. « Des décalages de distribution aux divergences d'acception ». In CONDAMINES, A. (dir.), Sémantique et Corpus. Hermès.

HABERT, B., A. NAZARENKO \& A. SALEM. 1997. Les linguistiques de corpus. Paris : Armand Colin.

HELGORSKY, F. 1981. «Les méthodes en histoire de la langue française. Évolution et stagnation ». Le Français Moderne 49/2, 119-144.

HILPERT, M. 2011. « Dynamic visualizations of language change: Motion charts on the basis of bivariate and multivariate data from diachronic corpora ». International Journal of Corpus Linguistics 16/4, 435-461.

HOUDEBINE, A-M. 1985. « Pour une linguistique synchronique dynamique ». La Linguistique 21, 7-36. HUMBERT-DROZ, J. 2017. « Définition d'un corpus comparable pertinent pour l'étude de la déterminologisation ». In Actes du colloque Jeunes Chercheurs - DyLis 2017, Diversité de la constitution des données : sur quoi travaille-t-on en Sciences du langage ?, Rouen (France).

HUMBERT-DROZ, J. En cours. « Circulation des termes entre langues de spécialité et langue générale : proposition d'un cadre théorique et méthodologique d'analyse du phénomène de la déterminologisation ». Thèse de doctorat en traduction et traitement informatique multilingue/ sciences du langage, Faculté de traduction et d'interprétation/Sciences du langage, Université de Genève/Université de Toulouse Jean-Jaurès (cotutelle).

HUMBLEY, J. 2011. « Vers une méthode de terminologie rétrospective ». Langages 183/3, 51-62.

JACQUES, M.-P. 2005. «L'emploi de termes réduits comme révélateur de la centralité dans le domaine ». In Actes de la conférence des 7èmes journées scientifiques du Réseau 'Lexicologie, Terminologie, Traduction' (Agence Universitaire de la Francophonie) : « Mots, termes et contextes ", Bruxelles (Belgique).

JOSEPH, B. \& R. JANDA (dir.). 2003. Handbook of Historical Linguistics. Oxford : Blackwell.

LAFRANCE, A. 2016. Étude de l'évolution dans la terminologie de l'informatique en anglais avant et après 2006. Maîtrise en traduction, Université de Montréal.

LERAT, P. 1995. Les langues spécialisées. Paris : Presses Universitaires de France.

LECOLLE, M. \& G. ACHARD-BAYLE (dir). 2009. Sentiment linguistique: Discours spontanés sur le lexique. Recherches Linguistiques 30 <halshs-00510859>. 
LECOLLE, M. (dir.). 2014. Métalangage et expression du sentiment linguistique « profane ». Le Discours et la langue $6 / 1$.

MAGNET, A. 2000. «Stratégies de validation du discours scientifique : Analyse diachronique du rôle de la section Discussion dans l'article de recherche ». ASp [En ligne] 27-30, mis en ligne le 01 janvier 2011. DOI : 10.4000/asp.2083.

MAIR, C. \& G. LEECH. 2006. « Current change in English syntax ». In AARTS, B. \& A. MACMAHON (dir.), The Handbook of English Linguistics. Oxford : Blackwell, 318-342.

MARCHELLO-NIZIA, C. 1995. L'évolution du français - Ordre des mots, démonstratifs, accent tonique. Paris : Armand Colin.

MARTINET, A. 1990. « La synchronie dynamique ». La Linguistique, Presses Universitaires de France $26 / 1,13-23$.

MEYER, I. 2001. « Extracting knowledge-rich contexts for terminography : A conceptual and methodological framework ». In BOURIGAULT, D., M.-C. L'HOMME \& C. JACQUEMIN (dir.), Recent Advances in Computational Terminology. Amsterdam/New York : John Benjamins Publishing Compan, 279-02.

MEYER, I. \& K. MACKINTOSH. 2000. « 'L'étirement' du sens terminologique : aperçu du phénomène de la déterminologisation ». In BÉJOINT, H. \& P. THOIRON (dir.), Le sens en terminologie. Lyon : Presses Universitaires de Lyon, 198-217.

MIKOLOV, T., K. CHEN, G. CORRADO \& J. DEAN. 2013. « Efficient estimation of word representations in vector space », consulté le 2 juillet 2018 <https://arxiv.org/abs/1301.3781/>.

MøLLER, B. 1994. « Où va la terminologie technique française? ». La Banque des mots 6 (Numéro spécial), 71-98.

MøLLER, B. 1998. « À la recherche d'une terminochronie ». Meta, Presses de l'Université de Montréal XLIII/3, 426-453.

NORMAND, C. 2000. Saussure. Paris : Les belles lettres.

PERUZZO, K. 2013. « Short-period evolution in EU legal texts: old and new terms, old and new concepts ». Linguistica 53/2,39-53.

PICTON, A. 2009. « Diachronie en langue de spécialité. Définition d'une méthode linguistique outillée pour repérer l'évolution des connaissances en corpus. Un exemple appliqué au domaine spatial ». Thèse de doctorat en Sciences du Langage, Université Toulouse Jean-Jaurès.

PICTON, A. 2014. « The dynamics of terminology in short-term diachrony: A proposal for a corpusbased methodology to observe knowledge evolution ». In TEMMERMAN, R. \& M. VAN CAMPENHOUDT (dir.), The Dynamics of Culture-bound Terminology in Monolingual and Multilingual Communication, Terminology and Lexicography Research and Practice. Amsterdam/Philadelphia : John Benjamins, 159-182.

РістоN, A. 2017. « Diatopy, diastraty and diachrony: Inputs from a 'dynamic' analysis of terms in a comparable specialised corpus ». In Actes de la conférence Language for specific purposes 2017, Interdisciplinary knowledge-making: challenges for LSP research Bergen (Norvège).

PICTON, A. \& P. DROUIN. 2017. « Linguistic approaches to track variation(s). An overview to (better) work with experts ». In Actes de la conférence Humanitarian Encyclopedia Scientific Committee meeting, Genève (Suisse). 
PICTON, A. \& P. DURY. 2015. « Les discours d'expertise en langues de spécialité : le point de vue du terminologue ». In BEAUDET, C. \& V. REY (dir.), Écritures expertes en questions. Aix-en-Provence : Presses universitaires de Provence, 265-278.

PICTON, A. \& P. DURY. 2017. « Diastratic variation in language for specific purposes. Observations from the analysis of two corpora ». In DRouin, P., A. FRANCOEUR, J. HUMBLEY \& A. PICTON (dir.), Multiple Perspectives in Terminological Variation. Amsterdam/New York : John Benjamins, 57-79.

QUEMADA, B. 1978. « Technique et langage, la formation des vocabulaires français des techniques ». In GILLE, B. (dir.), Histoire des techniques, techniques et civilisations, techniques et sciences. Paris : Gallimard, 1146-1240.

QUIRION, J. 2003. La mesure de l'implantation terminologique : proposition d'un protocole. Montréal : Office Québécois de la Langue Française.

RENOUF, A. 2017. « Some corpus-based observations on determinologisation ». Neologica 11, 21-48. RONDEAU, G. \& H. FELBER (dir.). 1981. Textes choisis de terminologie. : Québec : GIRSTERM, Université de Laval.

SIOUfFI, G., A. STEUCKARDT \& C. WIONET. 2012. « Comment enquêter sur les diachronies courtes et contemporaines? ». In Actes de la conférence $3^{e}$ Congrès Mondial de Linguistique Française, Lyon (France). DOI: <https://doi.org/10.1051/shsconf/2012010021>.

SOURNIA, J. C. 1994. « Les phases évolutives du vocabulaire médical français ». Meta, Presses de l'Université de Montréal XXXIX/4, 692-700.

STENGERS, I \& J. SCHLANGER. 1991. Les concepts scientifiques. Invention et pouvoir. Paris : Gallimard, Folio essais 161.

TARTIER, A. 2004. Analyse automatique de l'évolution terminologique : variations et distances. Thèse de doctorat en doctorat en informatique, Université de Nantes.

TEMMERMAN, R. 2000. Towards New Ways of Terminological Description. The Sociocognitive Approach. Amsterdam/Philadelphia : John Benjamins..

UNGUREANU, L. 2003. «L'interpénétration langue générale-langue spécialisée dans le discours d'internet ». Thèse de doctorat en Université Paris 13/Université Technique de Moldova.

VAN DER YEUGHT, M. 2009. Structurer l'anglais de la bourse : une approche des langues de spécialité par la spécialité. Habilitation à diriger les recherches, Bordeaux Université Victor-Segalen.

VERJANS, T. 2013. «Les locutions conjonctives. Une hypothèse romane ». In HADERMANN, P., Van

Acker M. \& B. MARIE-GUY (dir.), La variation et le changement en langue. Helsinki : Société Néophilologique , 133-147.

WOZNIAK, S. 2015. «Étude des domaines spécialisés et de leurs discours en diachronie : le cas des genres spécialisés de la littérature d'alpinisme aux États-Unis ». ASp [En ligne] 67, mis en ligne le 09 mars 2016. DOI : 10.4000/ asp.4659.

WÜSTER, E. 1981. «L'étude scientifique générale de la terminologie, zone frontalière entre la linguistique, la logique, l'ontologie, l'informatique et les sciences des choses ». In RONDEAU, G. \& H. FELBER (dir.), Textes choisis de terminologie. Québec, Canada : GIRSTERM, Groupe Interdisciplinaire de Recherche Scientifique et Appliquée en Terminologie. 


\section{NOTES}

1. Guespin (1995 : 206) remarque ainsi par exemple que « [1]a diachronie, que la théorie d'Eugen Wüster chasse par la porte, revient obstinément frapper au carreau, si fort même qu'elle ébranle la belle construction [...]».

2. Voir en particulier (Picton 2014 ; Condamines \& Dehaut 2011 ; Condamines \& Picton 2015 ; Picton 2014).

3. Voir (Condamines et alii 2018; Condamines \& Picton 2012; Condamines \& Picton 2014 ; Humbert-Droz en cours) pour une présentation de ces recherches.

4. Voir notamment (Meyer \& Mackintosh 2000 ; Galisson 1978; Renouf 2017 ; Ungureanu 2003) sur cette question.

5. Sur la base d'un calcul du $\chi 2$.

6. <http://smsc.cnes.fr/DEMETER/Fr/> consultée le 10 juin 2018, année du lancement du premier micro-satellite du Cnes Demeter.

7. (Picton $2009 ; 2014$ ).

8. (Condamines et Picton 2012 ; Condamines et alii 2018 ; Drouin, et alii 2018, à venir).

9. (Picton 2017).

10. Complémentarité révélée par exemple par certaines recherches qui s'interrogent sur le lien entre langue et science (notamment Stengers \& Schlanger 1991).

11. <https://humanitarianencyclopedia.org/>, consultée le 20 juin 2018.

12. Centre d'action et de recherche en action humanitaire de Genève, <https:// www.cerahgeneve.ch/home/> (consulté le 2 juillet 2018).

13. Nous détaillons ces approches dans (Drouin et alii 2018, à paraître).

\section{RÉSUMÉS}

Longtemps considérée comme le parent pauvre des recherches en langues de spécialité, la diachronie semble aujourd'hui prendre la place qui lui est due. Néanmoins, les travaux et recherches diachroniques présentent une grande hétérogénéité tant dans leurs approches que dans les objectifs qu'ils poursuivent. Si ceci reflète la richesse des apports de la diachronie, cela indique également que l'heure semble moins au plaidoyer en faveur de cette perspective, mais plus à la réflexion autour de ce qui la caractérise lorsqu'elle est appliquée aux langues de spécialité. Dans ce contexte, nous proposons d'adopter le prisme de la terminologie textuelle pour alimenter cette réflexion. Cette approche cherche en effet depuis plusieurs années à systématiser les méthodes outillées d'analyse de la variation en corpus spécialisés et à en théoriser différents aspects, à partir de contextes réels d'application. Ces objectifs ont donc amené ce courant à questionner plusieurs « piliers » d'analyse en diachronie autour des notions de corpus, outils, experts et interprétation. 


\section{INDEX}

Mots-clés : approche outillée, corpus, diachronie, terminologie textuelle, variation dialectale Keywords : Long neglected by research on specialized languages, diachronic approaches currently seem to experience their coming of age. However, studies on diachronic aspects of LSPs still show significant heterogeneity, both in objectives and methods. This may reflect their high investigative potential, but also supports the view that, while it is no longer necessary to make a plea for diachronic approaches, the ways in which they can be applied to LSPs warrant further investigation. We propose to adopt the angle of textual terminology to evaluate their applications in LSPs, as, for several years now, this approach has sought to build a systematic framework for tool-assisted methods aimed at analysing variations in specialized corpora, and has endeavored to establish their theoretical tenets, by factoring in actual contexts of use. In particular, textual terminology has questioned the validity of several "pillars" of diachronic studies, discussing such notions as "corpora," "tools, " "experts, " and "interpretation.

\section{AUTEUR}

\section{AURÉLIE PICTON}

Aurélie Picton est professeure associée au Département de traitement informatique multilingue (TIM) de la Faculté de traduction et interprétation de l'Université de Genève depuis 2011. Elle a obtenu un Doctorat en sciences du Langage en 2009, à l'Université de Toulouse 2, co-financé par le CNRS (laboratoire CLLE-ERSS [CNRS, UMR5263]) et le Centre national d'études spatiales (Cnes). Avant d'enseigner à Genève, elle a obtenu une bourse de recherche post-doctorale de la province du Québec (FQRNT) pour poursuivre ses recherches à l'Observatoire de linguistique Sens-Texte (OLST, Université de Montréal), puis a été ATER à l'Institut universitaire de formation des maîtres (IUFM Midi-Pyrénées). Ses principaux intérêts sont : la variation (en particulier diachronique) dans les langues de spécialité, les approches textuelles en terminologie, la linguistique de corpus et la linguistique outillée pour la traduction et la terminologie. Liste de publications récentes $<$ https://www.unige.ch/fti/fr/faculte/departements/dtim/membrestim/picton/>. Aurelie.Picton@unige.ch 